\title{
The Calculation of the Eigenvectors of a General Complex Matrix by Inverse Iteration
}

\author{
By J. M. Varah*
}

Introduction. In this paper, we are concerned with finding approximations to the eigenvectors of a general complex $n \times n$ matrix $A$, assuming good approximations to the eigenvalues have been found. In Section 1, we describe the method used, which amounts to one step of the well-known inverse iteration technique for a particular initial vector. We show that the eigenvector approximation thus obtained is as accurate as can be expected using single-precision arithmetic. An Algol 60 program using this method for the case of a general real matrix is given in the microfiche section, and the details of the program and an example are given in Section 2. In a second paper [7], we will describe a technique which gives rigorous a posteriori error bounds for such an approximate eigensystem, and which, applied repeatedly, can yield a better approximate eigensystem if the matrix $A$ is known to more than single precision.

1. The Basic Iteration. We are concerned with finding the column eigenvectors of a general complex $n \times n$ matrix $A$, that is, the columns of a matrix $X$ so that $A X=X \Lambda$, where $\Lambda$ is the diagonal matrix of eigenvalues $\left\{\lambda_{i}\right\}_{1}{ }^{n}$ of $A$. We assume the following:

(i) $A$ has been scaled so that $\|A\|_{2}=1$, for convenience.

(ii) $A$ has been reduced by a similarity transformation to upper Hessenberg form, i.e. $a_{i j}=0$ for $j \leqq i-2$. This reduction is usually done by Householder transformations or by elementary row and column operations. Both are discussed in Wilkinson [6, Chapter 6].

(iii) approximations $\left\{\lambda_{i}^{\prime}\right\}_{1}{ }^{n}$ to the eigenvalues of $A$ have been found, and are such that each is an exact eigenvalue of a slightly different matrix $A+E_{\lambda}$. The most popular eigenvalue method lately has been the $Q R$ method, which is known to give such approximate eigenvalues. This and other methods, including a Laguerre iteration developed by Parlett [3] are discussed in Chapters 7 and 8 of Wilkinson [6].

To find the eigenvectors, the inverse iteration technique has been very successful; namely we perform the following iteration for each eigenvalue approximation $\lambda$ :

$$
(A-\lambda I) y^{(k)}=y^{(k-1)}, \quad k=1,2, \cdots,
$$

with $y^{(0)}$ some arbitrary vector. The iteration is performed by first forming the $L U$ decomposition of $(A-\lambda I)$ using Gaussian elimination. That is, we form a unit lower triangular matrix $L$ and an upper triangular matrix $U$ such that $L U=$ $P(A-\lambda I)+F$, where $P$ is a permutation matrix and $F$ is a matrix of rounding errors. Then the two resulting triangular systems are solved.

It is our purpose here to show how the inverse iteration technique can provide

Received August 25, 1967. Revised February 23, 1968.

* Supported in part by the Office of Naval Research under contract Nonr-225(37) (NR044-211). 
as good an eigenvector approximation as can be expected. In fact, since $A$ is generally not represented exactly in the machine and because of rounding errors, all we can expect of an eigenvector approximation is that it can be an exact eigenvector of some matrix $A+E$, with $\|E\|_{2} \leqq c \eta_{1}$, where $c$ is close to 1 and

$$
\eta_{1}=\max \{\eta: f l(1+\eta)=1\} .
$$

Here $f(q)$ denotes the floating-point machine-calculated value of the arithmetic expression $q$.

Theorem 1. If at any step in the iteration (1), we find

$$
\frac{\left\|y^{(k)}\right\|_{2}}{\left\|y^{(k-1)}\right\|_{2}}>\frac{1}{r_{3}} \eta^{-1}
$$

then the computed vector $y^{(k)}$ is the exact eigenvector of a matrix $A+E$, where $\|E\|_{2} \leqq r \eta_{1}, r=r_{1}+r_{2}+r_{3}$, and $r_{1}$ and $r_{2}$ are small machine constants.

Proof. In the machine computation, since we use Gaussian elimination with partial pivoting, we know from Wilkinson [5, p. 98] that $P^{-1} L U=A-\lambda I+F$, where $\|F\|_{2} \leqq r_{1} \eta_{1}$. Moreover, in solving the triangular systems obtained, we know from Wilkinson [5, p. 102] that

$$
\left(P^{-1} L U+G_{k}\right) y^{(k)}=y^{(k-1)},
$$

where $\left\|G_{k}\right\|_{2} \leqq r_{2} \eta_{1}$. Here $r_{1}$ and $r_{2}$ depend on the machine arithmetic. Thus we have exactly

$$
\left(A-\lambda I+F+G_{k}\right) y^{(k)}=y^{(k-1)} .
$$

Now let $u=y^{(k)} /\left\|y^{(k)}\right\|_{2}$ and $v=y^{(k-1)} /\left\|y^{(k)}\right\|_{2}$. Then the above gives

$$
\left(A-\lambda I+F+G_{k}-v u^{*}\right) u=0 .
$$

Taking $E=F+G-v u^{*}$ gives

$$
\|E\|_{2} \leqq\left(r_{1}+r_{2}\right) \eta_{1}+\left\|y^{(k-1)}\right\|_{2} /\left\|y^{(k)}\right\|_{2}<r \eta_{1} .
$$

This completes the proof of the theorem.

So one strategy would be to use the basic iteration (1) and iterate until $\left\|y^{(k)}\right\|_{2} /\left\|y^{(k-1)}\right\|_{2}$ is close to $\eta_{1}^{-1}$. Wilkinson [6, Chapters 5 and 9] uses this technique, with $y^{(0)}=P^{-1} L e$ and $e=(1,1, \cdots, 1)^{T}$. He iterates until the norm ratio is close to $\eta_{1}{ }^{-1}$ and then does one more step. This strategy has two faults: first of all, we cannot guarantee that we will obtain a norm ratio close to $\eta_{1}^{-1}$. In fact, one can give examples where the iterates increase in norm by only $\eta_{1}^{-1 / n}$ at each step. In practice however, such a large norm ratio is usually obtained on the first iteration. But even so, for matrices with poorly conditioned eigenvalues, if we continue to iterate, the norm ratios are much less than $\eta_{1}^{-1}$ on each subsequent iteration, so even the "one more step" is not a good idea. We take a different viewpoint: we can guarantee a large norm on the very first iteration by choosing the initial vector properly.

Theorem 2. Let $\lambda$ be an approximate eigenvalue of $A$ with $\left(A-\lambda I+F_{1}\right)$ singular and $\left\|F_{1}\right\|_{2}=r \cdot \eta_{1}$, and let $P^{-1} L U$ be the $L U$ decomposition of $(A-\lambda I)$. Let $G$ be a matrix of linearly independent columns $\left\{g_{i}\right\}_{1}{ }^{n}$ and consider solving $U Z=G$ 
for the columns $z_{i}$ of $Z$. Suppose $\left\|z_{k}\right\|_{2} \geqq\left\|z_{i}\right\|_{2}, i \neq k$, and let $y^{(1)}$ be the computed solution to $U z_{k}=g_{k}$. Then $y^{(1)}$ satisfies

$$
\left\|y^{(0)}\right\|_{2} /\left\|y^{(1)}\right\|_{2} \leqq K \cdot \eta_{1}
$$

with

$$
K=n \sqrt{ } 2 \cdot \operatorname{cond}_{2}(G) \cdot\left[\left(\frac{n+1}{\sqrt{ } 2}\right)\left(r_{1}+r\right)+r_{2}\right],
$$

where $r_{1}$ and $r_{2}$ are given in Theorem 1. Thus $y^{(1)}$ is an exact eigenvector of $(A+E)$ with $\|E\|_{2} \leqq\left(K+r_{1}+r_{2}\right) \eta_{1}$.

Proof. Since $\left(A-\lambda I+F_{1}\right)$ is singular and $A-\lambda I=P^{-1} L U-F$, with $\|F\|_{2} \leqq r_{1} \cdot \eta_{1}$, then

$$
\left(P^{-1} L U-F+F_{1}\right) v=0
$$

for some vector $v$. Since $L$ and $U$ are both nonsingular,

$$
v=U^{-1} L^{-1} P\left(F-F_{1}\right) v,
$$

giving

$$
\left\|U^{-1}\right\|_{2}^{-1} \leqq\|P\|_{2}\left\|L^{-1}\right\|_{2}\left(r+r_{1}\right) \eta_{1} .
$$

Now since $A$ is in upper Hessenberg form, $L$ has only one nonzero element in each column below the diagonal, and because partial pivoting is used, this element is less than or equal to one in magnitude. Let $L_{k_{j}, j}$ be the nonzero off-diagonal element in the $j$ th column. Then solving for the $i$ th row of $L^{-1}$ (whose elements are denoted by $L_{i j}^{-1}$ ),

$$
\left|L_{i j}^{-1}\right|=\left|\sum_{k=j+1}^{i} L_{i k}^{-1} L_{k j}\right| \leqq\left|L_{i, k_{j}}^{-1} \cdot L_{k_{j}, j}\right|, \quad j=i-1, i-2, \cdots, 1 .
$$

We claim $\left|L_{i j}^{-1}\right| \leqq 1$ for all $i, j$. To see this, fix $i$. We proceed by induction on $j=i-1, i-2, \cdots, 1$, using the above equation. Now clearly $\left|L_{i, i-1}^{-1}\right|=$ $\left|L_{i, i-1}\right| \leqq 1$ and $L_{i i}^{-1}=1$. For $j<i-1$,

$$
\left|L_{i j}^{-1}\right| \leqq\left|L_{i, k_{j}}^{-1}\right| \cdot 1 \leqq 1
$$

using the induction hypothesis, since $k_{j}>j$. Thus

$$
\left\|L^{-1}\right\|_{2} \leqq(n(n+1) / 2)^{1 / 2}<(n+1) / \sqrt{ } 2,
$$

and

$$
\left\|U^{-1}\right\|_{2^{-1}} \leqq((n+1) / \sqrt{ } 2)\left(r+r_{1}\right) \eta_{1} .
$$

Now we are solving $U Z=G$ and hence

$$
\|Z\|_{2} \geqq\left\|U^{-1}\right\|_{2} /\left\|G^{-1}\right\|_{2} \text {. }
$$

Let the columns of $Z$ be $z_{i}, i=1, \cdots, n$, and suppose $\left\|z_{k}\right\|_{2} \geqq\left\|z_{i}\right\|_{2}, i \neq k$. Then

$$
\left\|z_{k}\right\|_{2} \geqq \frac{1}{\sqrt{ } n}\|Z\|_{2} \geqq \frac{1}{\sqrt{ } n} \frac{\left\|U^{-1}\right\|_{2}}{\left\|G^{-1}\right\|_{2}} .
$$

Now let our iterate $y^{(1)}$ be the computed approximation to $z_{k}$, that is, the machine 
approximation we would obtain solving for the right-hand side $g_{k}$. Then $y^{(1)}$ satisfies $\left(U+G_{1}\right) y^{(1)}=g_{k}$, with $\left\|G_{1}\right\|_{2} \leqq r_{2} \eta_{1}$, as in Theorem 1 . Hence

$$
\frac{1}{\left\|y^{(1)}\right\|_{2}} \leqq \frac{\left\|I+U^{-1} G_{1}\right\|_{2}}{\left\|z_{k}\right\|_{2}} \leqq \sqrt{ } n\left\|G^{-1}\right\|_{2}\left(\left\|U^{-1}\right\|_{2}^{-1}+r_{2} \eta_{1}\right) \text {. }
$$

When we solve for $y^{(1)}$ in this way, our initial vector $y^{(0)}=P^{-1} L g_{k}$, so that

$$
\left\|y^{(0)}\right\|_{2} \leqq\left\|P^{-1}\right\|_{2}\|L\|_{2} \cdot\|G\|_{2} \leqq(2 n)^{1 / 2}\|G\|_{2},
$$

and hence

$$
\frac{\left\|y^{(0)}\right\|_{2}}{\left\|y^{(1)}\right\|_{2}} \leqq n \sqrt{ } 2\left(\|G\|_{2} \cdot\left\|G^{-1}\right\|_{2}\right) \cdot\left[\left(\frac{n+1}{\sqrt{ } 2}\right)\left(r+r_{1}\right)+r_{2}\right] \eta_{1}=K \cdot \eta_{1} .
$$

Finally, applying Theorem 1, we see that $y^{(1)}$ is an exact eigenvector of $(A+E)$, with $\|E\|_{2} \leqq\left(K+r_{1}+r_{2}\right) \eta_{1}$. This completes the proof of Theorem 2 .

In effect, this just says that since $(A-\lambda I)$ is very nearly singular, the nearsingularity will be reflected in an inverse iterate with a large norm, for some initial vector. So in theory we could take any nonsingular $G$ and solve for each column. But this takes too much work: solving for every column takes $n^{3}$ operations. However, we do not have to find the column which gives the maximum solution norm, only one where the norm is large. So we want to use a $G$ in practice that gives a large norm for the very first column in most cases.

As Wilkinson noticed in [4], taking $G=I$ can be very poor. Here we are just solving for the columns of $U^{-1}$, and many of the matrices which arise have no small diagonal elements in $U$ although it is nearly singular. So it may be necessary to solve for several columns of $U^{-1}$ to find one with a large norm. Wilkinson has shown that the initial vector $e=(1,1, \cdots, 1)^{T}$ gives good results in practice; actually any such vector with lots of nonzero components would probably work as well. However, it can happen that $z=U^{-1} e$ is not large in norm either; we still must have other choices available.

The strategy we propose is to take for $G$ the orthogonal matrix

$$
g_{i j}=\cos \frac{2 \pi(i-1)(j-1)}{n}+\sin \frac{2 \pi(i-1)(j-1)}{n} .
$$

The first column is exactly the vector $e$ and in practice this almost always gives a solution with a large norm. If it does not however, we go on and try the second column, etc. Also note that $G^{T} G=n I$ so that $\operatorname{cond}_{2}(G)=1$ and thus this gives the best possible bound in Theorem 2 .

2. Description of the Program. The Algol 60 program, M-9, in the microfiche section herein, applies to a real matrix $A$ reduced to upper Hessenberg form. The reason for this is twofold: first, for an Algol compiler that allows complex declarations, the code for a general complex matrix is simpler than that given here because only the (a) iteration below is necessary. Secondly, if the matrix is real, time is saved by using a specialized program. For best results, the input matrix $A$ should be "balanced" so that its $i$ th row and column sums are roughly equal. This can be done by diagonal similarity transformations (see Osborne [2]). Also, the matrix should be scaled so that $\|A\|_{2}$ is close to 1.0 . 


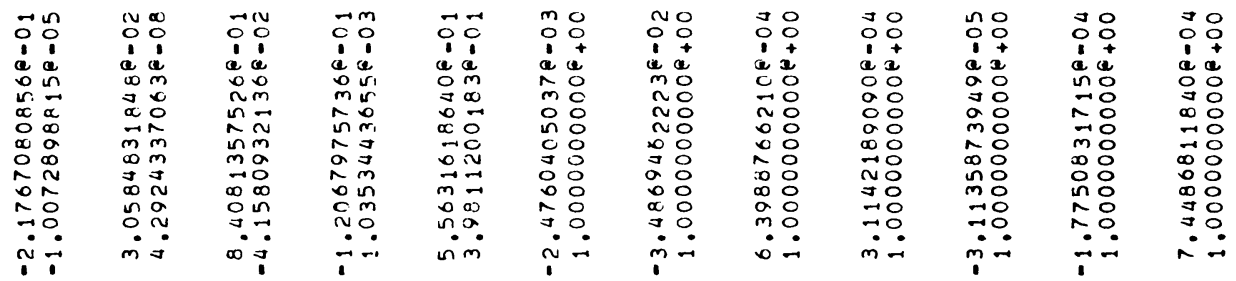

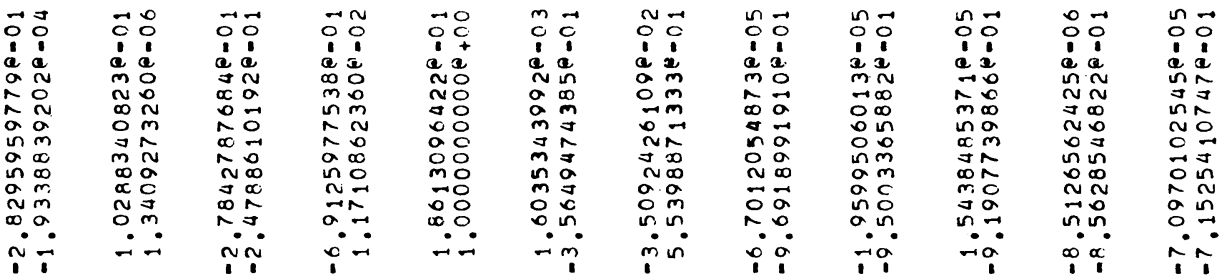

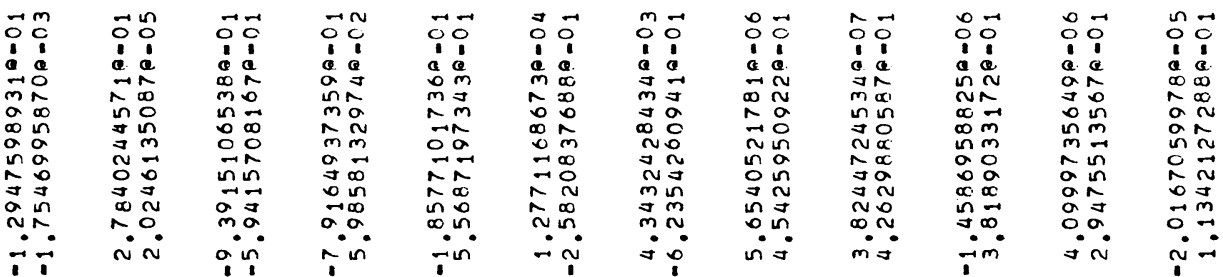

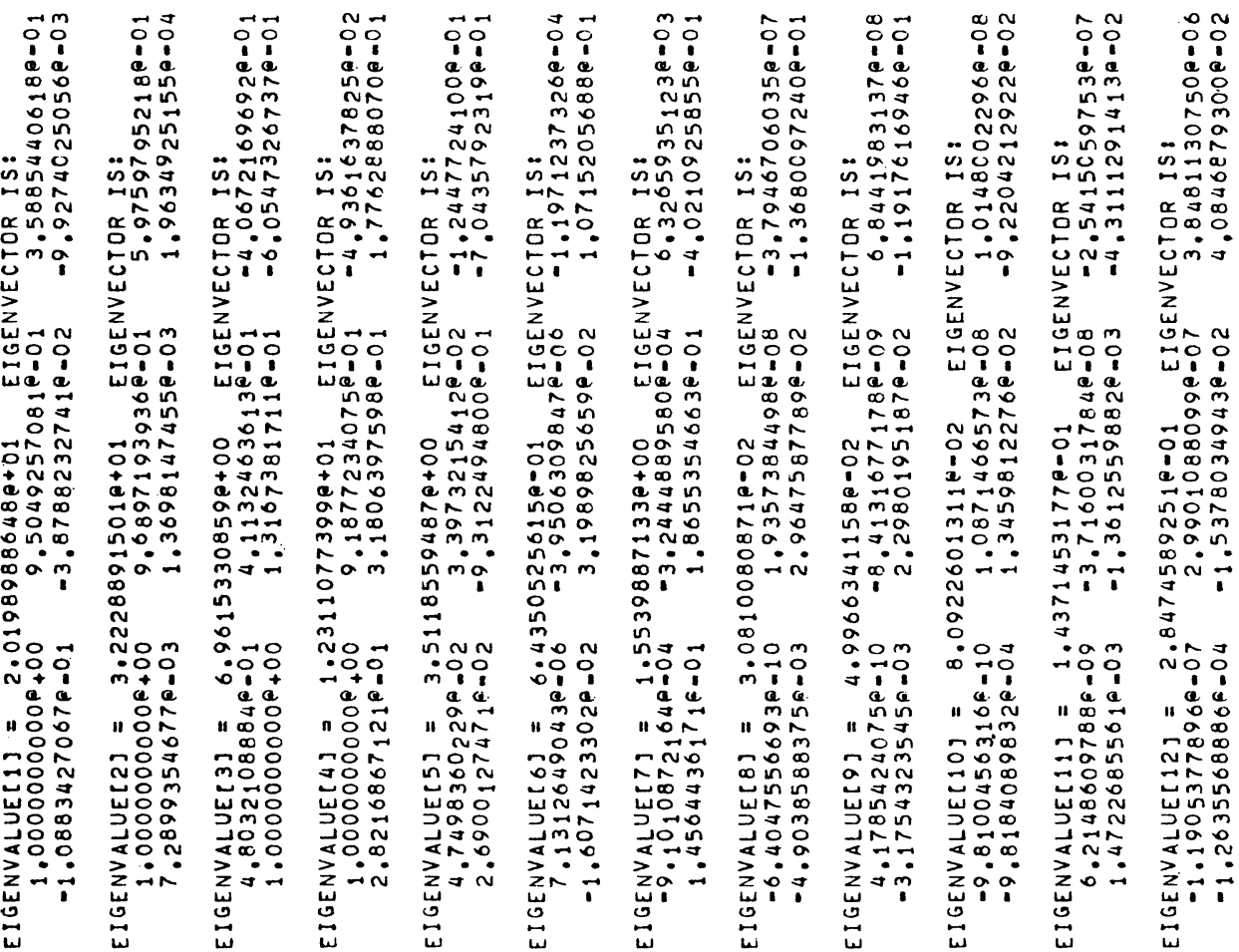


An Algol 60 program for the reduction to upper Hessenberg form using Householder transformations is given in [1] and a similar program using elementary similarity operations is forthcoming in Numerische Mathematik. An excellent program for calculating the eigenvalues by the $Q R$ method is forthcoming by Wilkinson in Numerische Mathematik, and the Algol 60 code for the Laguerre iteration of Parlett is given in [3].

The eigenvectors of the Hessenberg matrix are found as follows:

(a) if the eigenvalue approximation $\lambda$ is real, we form the $L U$ decomposition of $(A-\lambda I)$ and solve $U y^{(k)}=g_{k}, k=1,2, \cdots$, where the $g_{k}$ are the columns of $G$, defined by (2). We continue until $\left\|y^{(k)}\right\|_{2}>K \eta_{1}{ }^{-1}$, where $K$ is a fixed tolerance. The process requires about $n^{2}(k+1) / 2$ multiplications;

(b) if $\lambda=\xi+i \nu, \nu \neq 0$, we proceed as in Wilkinson [6, p. 630]. Since $\bar{\lambda}$ is also an eigenvalue approximation, we form the $L U$ decomposition of

$$
B=(A-\lambda I)(A-\bar{\lambda} I)
$$

and solve

$$
U y^{(k)}=g_{k}, \quad z^{(k)}=-\frac{1}{\nu}(A-\xi I) y^{(k)}, \quad k=1,2, \cdots .
$$

We continue until $\left\|y^{(k)}+i z^{(k)}\right\|_{2}>K \eta_{1}{ }^{-1}$. Since the matrix $A^{2}$ is formed only once, the work per eigenvalue is about $n^{2}(k+11 / 6) / 2$ multiplications.

If any zero pivots are encountered in the $L U$ decomposition, they are replaced by $\eta_{1} \max _{i, j}\left|a_{i j}\right|$, so that we can backsolve. If any eigenvalue approximations are identical, they are perturbed by an amount close to $\eta_{1}$ so that a different $L U$ is formed. Then if there are distinct eigenvectors corresponding to these eigenvalues, the two inverse iterations should give two distinct eigenvectors.

This program has been tested on scores of matrices using the Burroughs B5500 at Stanford University. We give one example:

$$
\begin{aligned}
a_{i j} & =n+1-\max (i, j), \text { if } j \geqq i-1 \text { and } n=12 . \\
& =0, \text { otherwise }
\end{aligned}
$$

This is the Frank matrix discussed by Wilkinson ([6, p. 92] and [5, p. 151]). It is in upper Hessenberg form and its eigenvalues are all real and positive. However, the Wilkinson conditions of the eigenvalues deteriorate as we go from biggest to smallest, and are about $10^{-7}$ for the last three eigenvalues. The eigenvalue approximations were found using an early version of Wilkinson's $Q R$ program on the B5500 where $\eta_{1}=\frac{1}{2} 8^{-12}$. The smallest three eigenvalue approximations are only correct to about two significant figures; nevertheless each is an exact eigenvalue of a slightly different matrix, as are the eigenvector approximations and each of the latter was found on the first inverse iteration.

Acknowledgment. The author would like to thank Professor G. E. Forsythe for his supervision and guidance, and Dr. J. H. Wilkinson for his many helpful suggestions. 
1. D. J. Mueller, "Householder's method for complex matrices and eigensystems of hermitian matrices," Numer. Math., v. 8, 1966, pp. 72-92. MR 33 \#872.

2. E. E. Osborne, "On pre-conditioning of matrices," J. Assoc. Comput. Mach., v. 7, 1960, pp. 338-345. MR 26 \#892.

3. B. N. Parlett, "Laguerre's method applied to the matrix eigenvalue problem," Math. Comp., v. 18, 1964, pp. 464-485. MR 29 \#2948.

4. J. H. Wilkinson, "The calculation of the eigenvectors of codiagonal matrices," Comput. $J .$, v. 1, 1958, pp. 90-96.

5. J. H. Wilkinson, Rounding Errors in Algebraic Processes, Notes on Applied Science No. 32, HMSO, London; Prentice-Hall, Englewood Cliffs, N. J., 1963. MR 28 \#4661.

6. J. H. Wilkinson, The Algebraic Eigenvalue Problem, Clarendon Press, Oxford, 1965. MR 32 \#1894.

7. J. M. VARAH, "Rigorous machine bounds for the eigensystem of a general complex matrix," Math. Comp., v. 22, 1968, pp. 793-801. 